\title{
Localización e Internet de las Cosas
}

\author{
Sosa, Eduardo Omar§; Godoy, Diego Alberto§ \\ $\S$ Secretaría de Investigación y Posgrado (SECIP). Facultad de Ciencias Exactas, Químicas y \\ Naturales - Universidad Nacional de Misiones - Argentina
}

Resumen. En este trabajo se presenta una arquitectura unificada para un sistema de localización basados en redes de sensores inalámbricos. Se presentan aquí dos escenarios de localización de objetos en IoT, uno interior y otro exterior, cada uno de ellos con sus técnicas asociadas. En el escenario interior se han desplegado nodos Decawave DWM1001, un nuevo producto de comunicaciones por radiofrecuencia de la familia Decawave ScenSor. El escenario seleccionado ha sido un aula de clases de la Facultad de Ciencias Exactas, Químicas y Naturales (FCEQyN) de la Universidad Nacional de Misiones (UNaM), utilizando métodos de comunicación de acuerdo a Ultrawideband (UWB) y Bluetooth Low Energy (BLE). En exteriores se han desplegado dispositivos basados en módulos XBee Pro S2 de $2.4 \mathrm{GHz}$ compatibles con ZigBee y IEEE 802.15.4 en una finca de producción ganadera cercana a la ciudad de Posadas de una superficie de 234 hectáreas $\left(2,34.10^{6} \mathrm{~m}^{2}\right)$. La localización de los nodos en los diferentes escenarios se ha calculado empleando el método de trilateración. En interiores se ha alcanzado sesgo aproximado de $7.10^{-2} \mathrm{~m}$; mientras que, en exteriores, el error calculado ha estado entre 35 m y $98 \mathrm{~m}$.

Palabras clave. IoT, Localización, Posicionamiento, Redes de Sensores

Abstract. A unified architecture for indoor and outdoor location determination in the IoT domain is presented in this work. Two empirical scenarios (indoor and outdoor) with their associated techniques have been proposed and successfully deployed. The selected physical location for interior experience has been a classroom of the Facultad de Ciencias Exactas, Químicas y Naturales (FCEQyN) from Universidad Nacional de Misiones (UNaM). The network has been set by deploying a set of Decawave DWM1001 nodes, a new radio frequency communications product from the Decawave ScenSor family that accomplish with communication methods as Ultrawideband (UWB) and Bluetooth Low Energy (BLE). For the outdoor scenario, a livestock production farm of 234 ha $\left(2,34.10^{6} \mathrm{~m}^{2}\right)$ near the city of Posadas has been selected. In this physical facility, devices based on $2.4 \mathrm{GHz}$ XBee Pro S2, compatible with ZigBee and IEEE 802.15.4, have been deployed. For the location process of the nodes, the trilateration method has been used. While in indoors essays an approximate bias of $7.10^{-2} \mathrm{~m}$ has been achieved, outdoors trilateration outcomes expose indeterminacy between $35 \mathrm{~m}$ and $98 \mathrm{~m}$.

Keywords: IoT, Location determination, Positioning, Sensor Networks 
Introducción. Internet de las cosas (IoT) es hoy la tecnología más prometedora, considerando el auge de los distintos objetos "inteligentes", que de alguna manera u otra estarán indefectiblemente presentes en la Red. El mencionado desarrollo ha despertado el nicho de investigación en la academia y la industria, como así también en las empresas, agencias gubernamentales y gobiernos [1]. La versatilidad de IoT permite que ésta utilice diversas tecnologías tales como comunicaciones inalámbricas, localización en tiempo real y redes de sensores. Esto permite integrar dispositivos físicos al mundo real de Internet. Desde 1998 [2] IoT ha impulsado la utilización de tecnología inalámbrica en dispositivos cotidianos, tales como electrodomésticos, receptores de audio/vídeo, detectores de humo, sistemas de refrigeración, etc. IoT pretende convertir a estos dispositivos en "Smart Things", dotándolos de capacidades de identificación, detección, interconexión y procesamiento; para así establecer vínculos con otros dispositivos y servicios a través de Internet, a través de la tecnología informática que permite la automatización de escenarios proporcionando una variedad de servicios convenientes y novedosos.

Las tecnologías inalámbricas penetran todos los aspectos de nuestras vidas. Internet de las Cosas y los Servicios Basados en Localización (LBS Location Based Services) son los pilares de los conceptos de ciudad inteligente (Smart City) y la inteligencia empresarial (Smart Business). 'Ciudad inteligente' se ha convertido en algo más que una palabra de moda en los últimos años. De hecho, con Internet de las cosas (IoT) y los dispositivos conectados, más ciudades de todo el mundo se están volviendo más inteligentes que nunca. Las "cosas" inteligentes de IoT son una parte indivisible de Internet; ello como consecuencia de la capacidad de los nodos (de la red de sensores inalámbricos) de calcular y comunicarse. La necesidad de ubicación física de los nodos se presenta en todas las capas, desde el nivel de la aplicación (para interpretar correctamente las mediciones realizadas por los nodos desplegados), hasta el nivel físico (para detectar la cobertura).

El servicio basado en la ubicación pasa a ser un servicio fundamental, mientras que la precisión de la localización es un problema clave. Las tecnologías ideales para usuarios de IoT son aquellas de configuración mínima y de utilización inmediata. IOT hace uso de tecnologías para obtener información de localización física. Las tecnologías de radio más comunes (por ejemplo, ZigBee [3], Bluetooth [4]) no están adaptadas para escenarios de largo alcance, mientras que soluciones basadas en comunicaciones celulares $(2 \mathrm{G}, 3 \mathrm{G}, 4 \mathrm{G}$ y $5 \mathrm{G})$ pueden proporcionar una mayor cobertura, pero consumen energía de forma excesiva.

El sistema de Posicionamiento Global (GPS) es una tecnología madura y confiable, que se adapta a un gran alcance, por lo que es ampliamente utilizada en localizaciones en exteriores. GPS es aplicado a sistemas inteligentes de transporte (ITS), navegación, peaje, estacionamiento y la prevención de colisiones; pudiendo alcanzarse una precisión cercana a los 5 metros [4]. Sin embargo, GPS carece de la eficiencia y probada exactitud cuándo se utiliza en ambientes interiores. La desventaja de GPS es el excesivo consumo de energía de los nodos, por lo cual no es recomendable en los entornos de IoT. 
Los sistemas de localización se incorporaron a varios sistemas, i.e. localización de robots [5], [6]; aplicaciones en mercados [7], posicionamiento IEEE 802.11 [8], sistemas basados en el tiempo [9] y varios más. Sin embargo, todos los sistemas de ubicación comparten conceptos y tareas comunes. Cada sistema de ubicación requiere algún tipo de detección / medición de señal de un parámetro que depende de la ubicación. En un modelo unificado es importante que se admita una amplia gama de tecnologías, incluso múltiples tecnologías simultáneamente. Dichas tecnologías se incorporan a un sistema complejo que definimos como proveedor de posición, el cual proporciona una abstracción para la capa de middleware.

La capa Middleware, que hace de interfaz entre el Hardware que subyace en la capa de Posicionamiento y el LBS está basada en [10] y es responsable de la integración(Capa de integración) de los proveedores de múltiples posiciones, como la fusión de datos, formatos y contexto.. Finalmente, la capa de aplicación realiza procesamientos adicionales a nivel aplicación y usuario de la información de ubicación para que pueda ser aprovechada por el LBS. La Figura 1 muestra la propuesta de arquitectura completa [11]

Se han propuesto diferentes técnicas, tecnologías inalámbricas y mecanismos en la literatura para proporcionar servicios de localización en interiores con el fin de mejorar los servicios prestados a los usuarios como el ángulo de llegada (AoA), la potencia de señal recibida (RSS) y el tiempo de vuelo (ToF), el tiempo de vuelo de retorno (RTOF) y entendiéndose como ToF a la técnica utilizada para estimar distancias de cuerpos calculando el tiempo transcurrido entre la emisión y la recepción de la señal emitida [12].

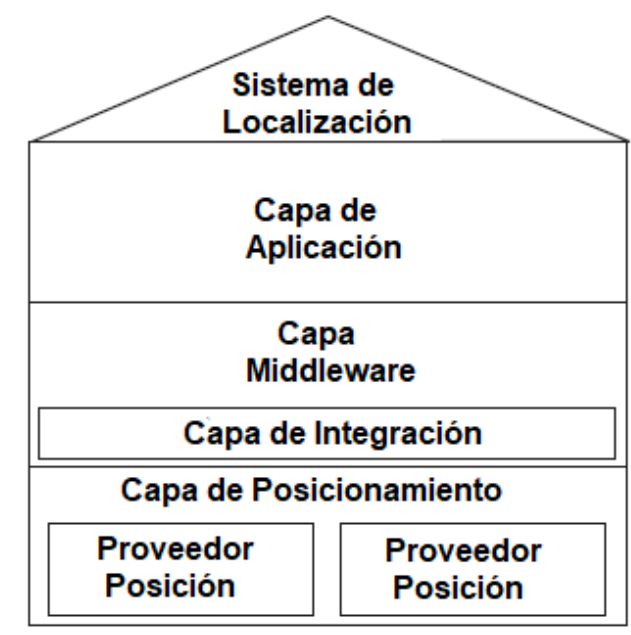

Figura 1. Propuesta unificada para la arquitectura de un sistema de localización (Elaboración Propia)

El interés empresarial de aplicaciones y servicios referentes a la ubicación ha ido aumentado a lo largo del tiempo, por lo que la demanda de servicios precisos de localización en interiores se ha 
vuelto importante. Al día de hoy, se están realizando investigaciones relacionadas con sistema de posicionamiento interior (IPS) en ámbitos académicos e industriales [13] [14].

Las técnicas existentes para la localización en interiores y exteriores poseen baja exactitud o son demasiado complejas, requiriendo una alta inversión. En el presente trabajo evaluamos técnicas de moderada precisión, cumpliendo con los requisitos de baja complejidad y costo.

\section{Materiales y Métodos}

Se presentan en éste manuscrito dos escenarios de localización de objetos: interior y exterior.

Desde hace un tiempo existen sistemas de localización en interiores basados en Ultrawideband (UWB), sistema que proporcionan una alta precisión a bajo costo, lo cual es muy conveniente particularmente en IoT. UWB es una tecnología de radiofrecuencia (RF) capaz de ofrecer transmisión de datos de alta velocidad (hasta 400 ó $500 \mathrm{Mbit} / \mathrm{s}$ ) y baja potencia; en distancias de unos pocos metros. Su principal ventaja respecto a otras tecnologías inalámbricas radica en el hecho de que puede transmitir más datos utilizando menos potencia que el resto de sistemas disponibles [15]. Adicionalmente, los equipos de radio necesitan menos componentes, por lo que se convierte en una solución económica.

Normalmente UWB implementa información de distancia basada en mediciones de tiempo de vuelo (ToF), estimando la ubicación de los objetos mediante un algoritmo particular. Sin embargo se presentan problemas con las mediciones de distancia a puntos sin línea de visión (NLOS Non- Line-of-Sight) debido a la propagación por trayectos múltiples que conduce estimaciones de distancia incorrectas [16].

Los sistemas de localización de UWB han mostrado históricamente una buena precisión en determinación de localización de objetos, siendo ésta superior a la de todas las técnicas de banda estrecha. De todas maneras, la tecnología no ha despertado un interés generalizado. A diferencia de las técnicas de banda estrecha, los sistemas UWB son intrínsecamente difíciles de diseñar, ya que los circuitos de temporización requieren un diseño personalizado del circuito integrado [17], o como en el caso del presente trabajo, utilizar un kit listo para usar.

El Kit Módulo de evaluación y desarrollo 1001 de Decawave ${ }^{\circledR}$ se basa en el circuito integrado DW1000, el cual proporciona un transceptor UWB con todas las primitivas de localización integradas. El transceptor puede proporcionar marcas de tiempo para los paquetes entrantes y las mediciones de tiempo de vuelo: dos primitivas necesarias para los sistemas de ubicación basados en tiempo, permitiendo así la investigación futura en los sistemas de ubicación UWB. La Figura 2a muestra uno de los nodos de nuestro despliegue, capaz de funcionar como ancla o móvil.

Para localización en exteriores, se ha desplegado una red a cielo abierto, implementando un nodo coordinador, tres o más nodos ancla (fijos) y diferentes nodos móviles (itinerantes). Los diferentes nodos son provistos por Digi International, siendo modelo XBee Pro S2 de $2.4 \mathrm{GHz}$ compatibles con ZigBee y IEEE 802.15.4 [3] (Figura 2b). Los nodos ancla están vinculados a módulos Raspberry PI, de 256 MB de memoria RAM, siendo los mismos geo localizados por 
medio de GPS. La distancia existente entre cada ancla y su altura respecto al suelo se ha determinado por medio de Radio Mobile [20].

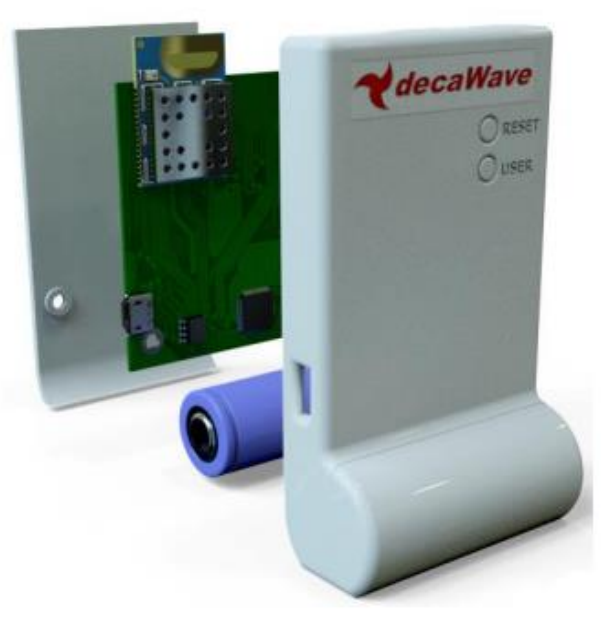

a)

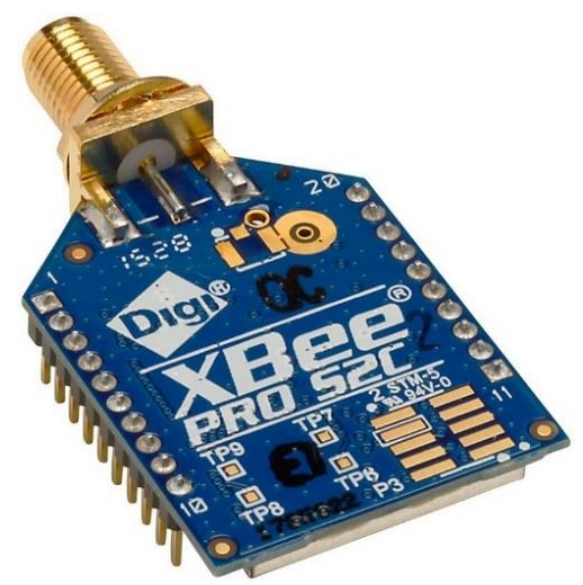

b)

Figura 2. a) Nodo de MDEK 1001 utilizado en localización en interiores [18], b) Tranceptor XBee Pro S2 utilizado en localización exterior [19].

Con la captura de paquetes de datos, se han efectuado los cálculos de atenuación de la señal (RSSI). Si las tramas recibidas son válidas, las mismas se almacenan en una base de datos. Toda esta información se presenta en una interfaz gráfica, la cual se actualiza automáticamente en cada recepción. En la etapa de trilateración [21], el sistema verifica que haya al menos tres nodos anclas hayan capturado el RSSI del nodo móvil para realizar el cálculo correspondiente (Figura $3)$.

\section{Resultados}

\section{a) Localización interior}

Los resultados de la experiencia se obtuvieron con un kit de desarrollo para el módulo DWM1001 (ver Fig, 2) de Decawave DWM1001 [18]. Es un producto de la línea de productos de comunicaciones por radiofrecuencia Decawave ScenSor, Es un circuito integrado de ultra ancho de banda (UWB) de baja potencia, de un solo chip IEEE 802.15.4-2011, radio BLE (Bluetooth Low Energy), precargado con un firmware de sistema de localización en tiempo real (RTLS) certificado. 


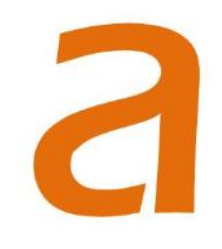

TAS Journal, vol. 4, n. 3, p. $146-157$

ISSN 2595-1521

SEPTEMBER 2018

eososa@unam.edu.ar

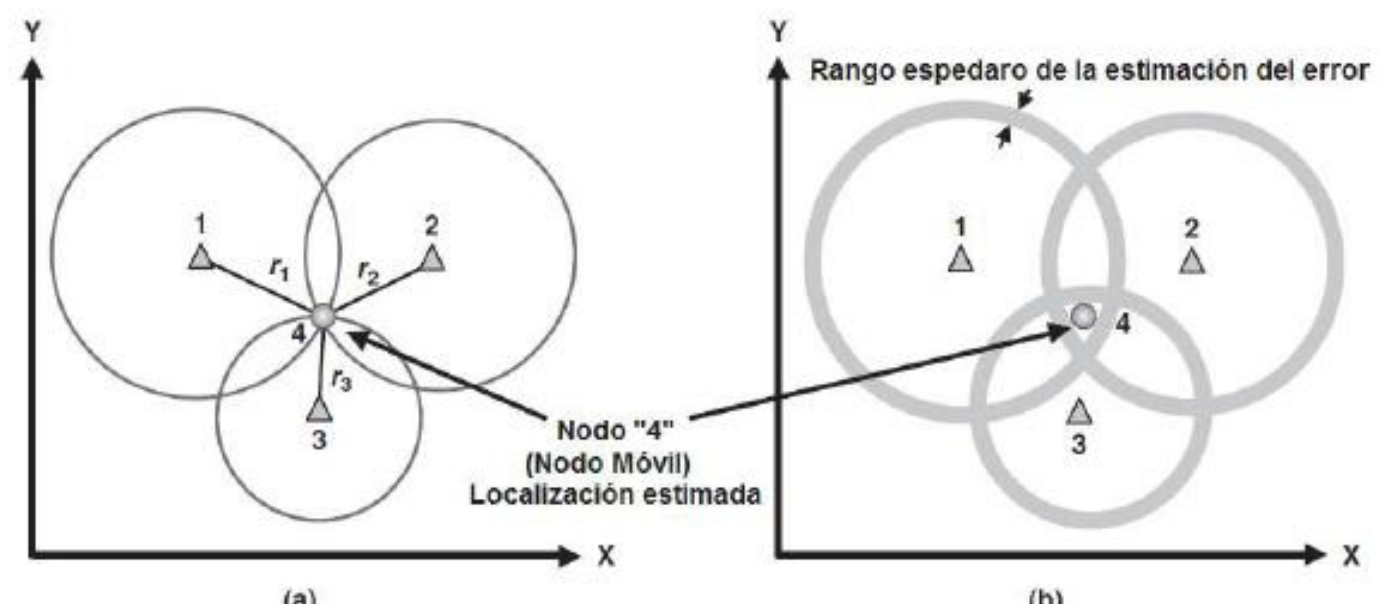

(a)

(b)

Figura 3. Localización de nodos por trilateración a) Ideal ; b) con error asociado [22].

Cada uno de los nodos provee los siguientes elementos; tranceptor UWB DW1001 (compatible IEEE802.15.4-2011), antena PCB UWB (6.5 GHz), velocidad máxima de transferencia de datos 6.8 Mbps, alcance en línea de visión aproximadamente $60 \mathrm{~m}$, nRF52832 system on chip $2,4 \mathrm{GHz}$, capaz de transferir a velocidades 2 Mbps en modo Bluetooth low energy - ARM Cortex M4, 512K Flash; antena de Bluetooth.

Los datos de las experiencias han sido obtenidos de experiencia y despliegues realizados en el Aula 12 (salón de dictado de clases) de la Facultad de Ciencias Exactas, Químicas y Naturales, con una superficie aproximada de $110 \mathrm{~m}^{2}(13,30 \mathrm{~m} \times 8,30 \mathrm{~m})$, libre de obstáculos y objetos que pudieran provocar atenuación de las señales radioeléctricas (figura 5 a).

En el aula se han distribuido 4 nodos ancla, realizando la calibración de del sistema de manera automática. El posicionamiento automático es una configuración del kit utilizado para determinar automáticamente las ubicaciones de los anclajes. Debe considerarse que esta característica puede provocar un pequeño error en la ubicación de los nodos ancla, lo que hace que las ubicaciones informadas por el sistema sean menos precisas.

El resultado de una de las experiencias de la localización de un nodo en el Aula 12 se muestra en la Figura 5 b. Comprobada la posición del nodo DW0A3B por medio de EDM (Electronic Distance Measurement) la indeterminación del método alcanza los $7.10^{-2}$ metros.

\section{b) Localización exterior}

En éste escenario se pretende el seguimiento y la localización de nodos móviles por medición del nivel de potencia de las señales recibidas (RSSI) por los dispositivos (nodos sensores) en la red inalámbrica.. 
Para este caso se han seleccionado escenarios con una cantidad de árboles de moderada a abundante, como también escenarios de terreno irregular. El posicionamiento de los nodos ancla considera la no linealidad entre ellos, debiendo ser la potencia de la señal tal que asegure la comunicación entre los mismos.

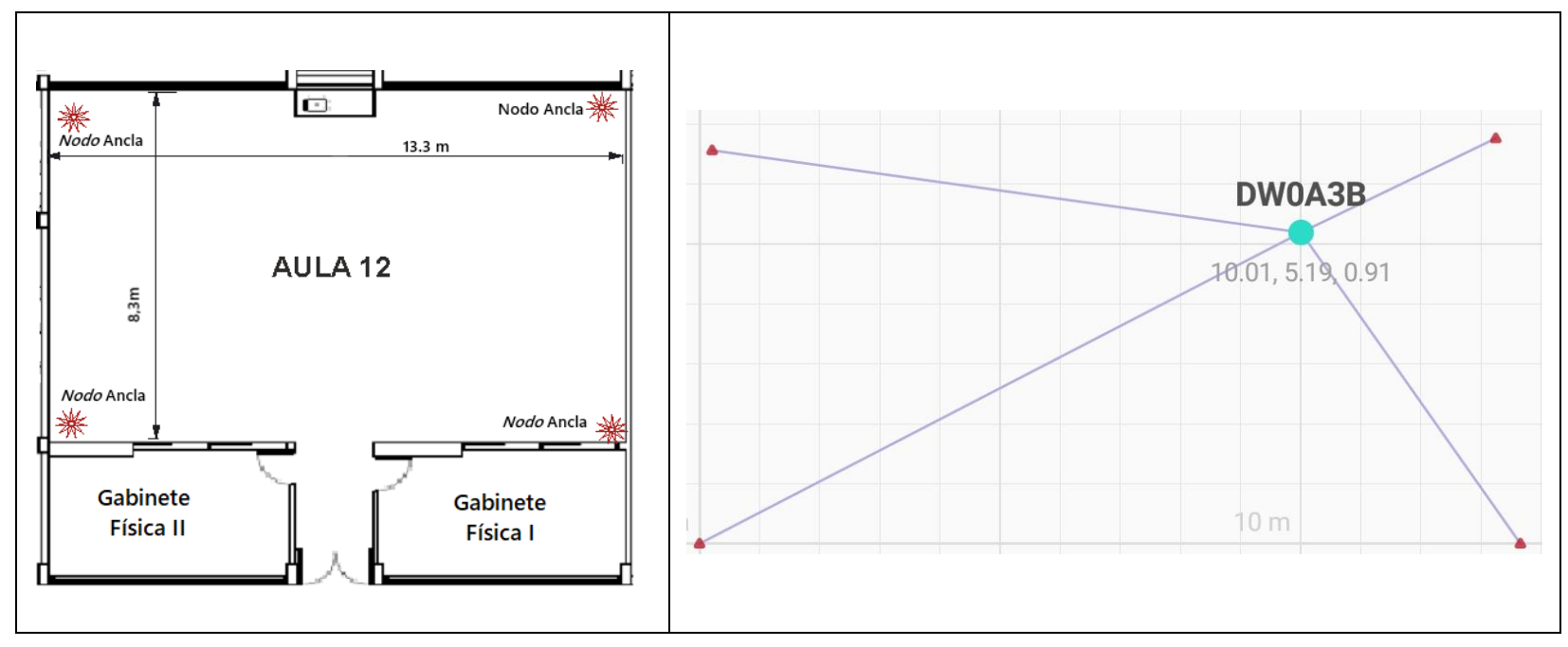

c)

b)

Figura 5. a) Escenario de localización Interior, b) resultado localización de un nodo en Aula 12. (Elaboración Propia)

La parametrización de la altura de las antenas se ha realizado en base a los niveles de la potencia de la señal recibida. De ésta manera la aplicación Radio Mobile permite establecer el nivel de potencia de recepción entre los nodos ancla y nodos móviles considerando solamente la elevación del terreno (Tab, 1), logrando con ello evitar considerar la atenuación producida en los diferentes escenarios. Para validar los modelos utilizados, es necesario de cualquier manera, calibrar el sistema determinando el coeficiente de atenuación en las diferentes zonas. El área global de desarrollo de la experiencia ha sido de 234 hectáreas $\left(2,34.10^{6} \mathrm{~m}^{2}\right)$, ver Figura 6 .

Tabla 1. Altura promedio de los nodos ancla (Nodos Fijos)

\begin{tabular}{lcc}
\hline & Terreno con vegetación & Terreno irregular \\
\hline Nodo ancla 1 & 10.75 & 17.50 \\
Nodo ancla 2 & 14.50 & 15.60 \\
Nodo ancla 3 & 8.16 & 17.83 \\
\hline
\end{tabular}


La zona 1 se establece en una zona arbolada, mientras que la zona 2 se establece en terreno irregular, definiendo luego la geoposición global de cada uno de los nodos.

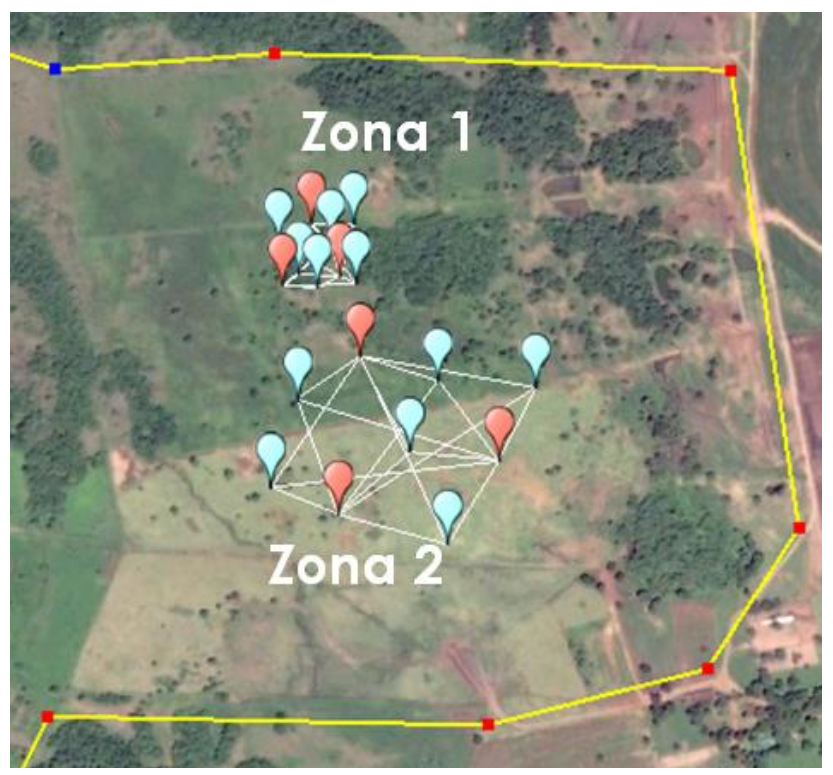

Figura 6. Área de búsqueda nodos móviles (Elaboración Propia)

Para determinar el coeficiente de atenuación, se han realizado mediciones cada $5 \mathrm{dBm}$ de potencia de señal. (Tabla 2), donde se establece el valor del coeficiente de atenuación $\boldsymbol{n}$ de 0.96 . valor para el cual la indeterminación es de $9.10^{-2}$ metros.

En las zonas con vegetación se han considerado los modelos de propagación Weissberger [23], y los modelos de Atenuación debida a la vegetación de la Unión Internacional de Telecomunicaciones (UIT) [24] y el modelo UIT Temprano ${ }^{1}$. En las zonas de terreno irregular si analizarán los modelos Egli y UIT [25].

Tabla 2. Error mínimo para cada coeficiente $n$

\begin{tabular}{cccc}
\hline $\begin{array}{c}\text { Nro } \\
\text { Prueba }\end{array}$ & $\begin{array}{c}\text { RSSI } \\
\text { dBm }\end{array}$ & $\begin{array}{c}\text { coeficiente } \\
\text { de atenuacion }\end{array}$ & $\begin{array}{c}\text { Error } \\
\text { metros }\end{array}$ \\
\hline 1 & -45 & 0.30 & 0.24 \\
2 & -50 & 0.53 & 0.19 \\
3 & -55 & 0.74 & 0.35 \\
4 & -60 & 0.96 & 0.09 \\
\hline
\end{tabular}

\footnotetext{
${ }^{1}$ Modelo adoptado en 1986 por el Comité Consultivo Internacional de Radiocomunicaciones (CCIR) [28]
} 


\begin{tabular}{cccc}
\hline $\begin{array}{c}\text { Nro } \\
\text { Prueba }\end{array}$ & $\begin{array}{c}\text { RSSI } \\
\text { dBm }\end{array}$ & $\begin{array}{c}\text { coeficiente } \\
\text { de atenuacion }\end{array}$ & $\begin{array}{c}\text { Error } \\
\text { metros }\end{array}$ \\
\hline 5 & -65 & 1.16 & 0.91 \\
6 & -70 & 1.35 & 1.66 \\
7 & -75 & 1.49 & 2.05 \\
8 & -80 & 1.65 & 4.71 \\
9 & -85 & 1.79 & 6.80 \\
10 & -90 & 1.93 & 2.49 \\
11 & -95 & 2.08 & 3.43 \\
12 & -100 & 2.24 & 10.03 \\
\hline
\end{tabular}

Los resultados de la zona 1 se muestran en la Tab. 3. El modelo de propagación Weissberger, localiza 2 de 6 nodos móviles. El modelo de propagación UIT Temprano encuentra 5 de los 6 nodos móviles, mientras que el modelo de propagación UIT, encuentra 1 de 6 nodos móviles. El error promedio es de 35.55 metros y el error mínimo es de 7.80 metros.

Tabla 3. Comparación de cada modelo de propagación en zona 1

\begin{tabular}{lc}
\hline Modelo & $\begin{array}{c}\text { Nodos localizados/total } \\
\text { desplegados }\end{array}$ \\
\hline Weissberger & $2 / 6$ \\
UIT Temprano & $5 / 6$ \\
UIT & $1 / 6$ \\
\hline
\end{tabular}

Igualmente, para terreno irregular (zona 2) se localizan según Egli 16 nodos móviles, mientras que el modelo de propagación UIT localiza a todos los nodos móviles. El error promedio en éste caso es de 98,18 metros y el error mínimo es de 11,66 metros.

Conclusiones y Trabajos Futuros. En éste documento presentamos alternativas de utilización de tecnologías de radiofrecuencia para localización de objetos en el dominio de Internet de las Cosas. El sistema es capaz de llevar a cabo su tarea de forma continuada localizando los nodos en interiores o exteriores.

La utilización de los sistemas existentes de detección y localización de ubicaciones interiores en un entorno doméstico ha estado limitada hasta el presente debido al elevado costo de la tecnología y a la baja precisión de la estimación de la ubicación. El sistema propuesto ha sido validado para detectar y rastrear ubicaciones en interiores y campo abierto de manera precisa, utilizando una plataforma de localización inteligente que utiliza la tecnología UWB (IEEE 802.15.4), y BLE; y nodos XBee compatibles con ZigBee y IEEE 802.15.4. La mayor precisión, introducida por este enfoque, hace que la localización sea más confiable.

Si bien la exactitud alcanzada en localización interior parece adecuada, se realizarán mejoras al método apuntando a mejores resultados, Para ello se establecerán las posiciones de anclaje con 
exactitud por medio de EDM (Electronic Distance Measurement) ingresándolas al sistema manualmente. Asimismo, el KIT MDEK1001 nos permite evaluar otras configuraciones, por ejemplo: 11 Anclas + 1 Nodo; 4 Anclas + 8 Nodos; y 4 Anclas +7 Tags+1 Listener.

Sabido es que la localización en ambientes al aire libre se realiza tradicionalmente a través de los sistemas de posicionamiento global (GPS Global Positioning System), cuya precisión alcanza cerca de 5 metros. La desventaja de la implementación de GPS es el excesivo consumo de energía, no siendo recomendables en los entornos de IoT. La precisión alcanzada en ambientes exteriores no ha satisfecho nuestras expectativas; por ello en un futuro cercano se considera la utilización de tecnologías que se adaptan a las necesidades y demandas de entornos IoT. Estas tecnologías son las LPWAN (Low Power Wide Area Network) [26],la que complementa, y ciertas veces sustituyen a las tecnologías inalámbricas existentes para diversas aplicaciones emergentes, sea el alcance que fuere considerado [27].

Reconocimiento: El presente trabajo y manuscrito, se ha desarrollado en el marco del proyecto "Localización de Objetos en Internet de las Cosas", acreditado bajo código 16Q652-PI en la Secretaría de Investigación y Posgrado de la Facultad de Ciencias Exactas, Químicas y Naturales, Universidad Nacional de Misiones.

Declaración. En el presente trabajo, los autores no declaran conflicto de intereses alguno.

\section{Referencias}

[1] M. Feki, F. Kawsar, M. Boussard y L. Trappeniers, «The Internet of Things: The Next Technological Revolution,» Computer, vol. 46, nº 2, pp. 24-25, 2013.

[2] K. Ashton, «That 'Internet of Things' Thing,» 2009. [En línea]. Available: http://bit.ly/2LnVOaW.

[3] ZigBee Alliance Specifications, «"802.15.4 vs ZigBee",» [En línea]. Available: http://www.libelium.com/es/802-15-4-vs-zigbee/.

[4] V. Cantón Paterna, A. Calveras Augé, J. Paradells Aspas y M. Pérez Bullones, «A Bluetooth Low Energy Indoor Positioning System with Channel Diversity, Weighted Trilateration and Kalman Filtering,» Sensors, vol. 17, nº 12, p. 2927, 2017.

[5] N. Drawil, H. Amar y O. Basir, «GPS Localization Accuracy Classification: A Context-Based Approach,» IEEE Transactions on Intelligent Transportation Systems, vol. 14, nº 1, Marzo 2013.

[6] F. Boniardi, A. Valada, R. Mohan, T. Caselitz y W. Burgard, «Robot Localization in Floor Plans Using a Room Layout Edge Extraction Network,» arXiv:1903.01804, 2019.

[7] M. Hamer y R. D’Andrea, «Self-Calibrating Ultra-Wideband Network Supporting Multi-Robot 
Localization,» vol. 6, pp. 22292-22304, 2018.

[8] Z. Wu y F. Jia, «Toward a theory of supply chain fields - understanding the institutional process of supply chain localization,» Journal of Operations Management, Vols. \%1 de \%258-59, pp. 27-41, 2018.

[9] A. Salman, S. El-Tawab, Z. Yorio y A. Hilal, «Indoor Localization Using 802.11 WiFi and IoT Edge Nodes,» de memories IEEE Global Conference on Internet of Things (GCIoT), Alejandria, Egypt, 2018.

[10] M. Ibrahim, T. M. y M. ElNainay, «, "CNN based Indoor Localization using RSS Time-Series,» de IEEE Symposium on Computers and Communications (ISCC), Natal, 2018.

[11] D. A. Godoy, R. Díaz Redondo y E. O. Sosa, «Internet De Las Cosas: Middleware De Gestión De Datos De WSN,» de WWW/INTERNET 2015 e COMPUTAÇÃO APLICADA, Florianòpolis, 2015.

[12] M. Pelka y H. Hellbrück, «Survey of challenges and towards a unified architecture for location systems,» Journal of Network and Computer Applications, vol. 67, pp. 75-85, 2016.

[13] F. Zafari, A. Gkelias y K. Leung, «A Survey of Indoor Localization Systems and Technologies,» IEEE Communications Surveys \& Tutorials, 2019.

[14] Y. Chen, T. Li, Y. Cui, Z. Li, M. Gao y L. Zhou, «Research on Indoor and Outdoor Integrated Location Service Technology,» de 2018 International Conference on Intelligent Transportation, Big Data \& Smart City (ICITBS), Xiamen, 2018.

[15] H. Huang, Q. Zeng, R. Chen, Q. Meng, J. Wang y Z. Zeng, «Seamless Navigation Methodology optimized for Indoor/Outdoor Detection Based on WIFI,» de Ubiquitous Positioning, Indoor Navigation and Location-Based Services (UPINLBS), Wuhan, 2018, 2018.

[16] Grupo ADSL Zone, «¿Qué es? UWB (Ultra Wide-Band),» 2006. [En línea]. Available: http://bit.ly/2luTyFG.

[17] M. Pelka, P. Bartmann, S. Leugner y H. Hellbrück, «Minimizing Indoor Localization Errors for NonLine-of-Sight Propagation (ICL-GNSS),» de Memories of 8th International Conference on Localization and GNSS, Guimaraes, 2018.

[18] B. Campbell, P. Dutta, B. Kempke, Y. Kuo y P. Pannuto, «DecaWave: Exploring State of the Art Commercial Localization,» Electrical Engineering and Computer Science Department - University of Michigan, 2017.

[19] Simmetry Electronics, «DWM1001 ScenSor,» 2019. [En línea]. Available: http://bit.ly/2L2tWdP. [Último acceso: Agosto 2019].

[20] Digi International, «"XBee / XBee-PRO - ZigBee RF Modules",» 2015. [En línea]. Available: ftp://ftp1.digi.com/support/documentation/90000976.pdf. [Último acceso: 2015]. 
[21] Radio Mobile, «Radio Mobile - RF propagation simulation software,» mayo 2019. [En línea]. Available: http://radiomobile.pe1mew.nl/.

[22] E. García, «Técnicas de Localización en Redes Inalámbricas de Sensores.,» Researchgate.net, [En línea]. Available: http://bit.ly/2ly9I0U. [Último acceso: Junio 2019].

[23] Waltenegus Dargie, Christian Poellabauer, "Fundamentals of Wireless Sensor Networks: Theory and Practice". ISBN: 978-0-470-99765-9, Editorial Willey, 2011.

[24] O. Kurnaz y H. Selcuk, «Near ground propagation model for pine tree forest environment,» $A E U$ International Journal of Electronics and Communications, vol. 68, $\mathrm{n}^{\circ}$ 10, pp. 944-950, 2014.

[25] Unión Internacional de Telecomunicaciones (UIT), «Recomendación UIT-R P.833-9 - Atenuación debida a la vegetación,» 2016.

[26] M. Mahrokh, E. Mehrshahi y K. Sandrasegaran, «Accurate RSS-Based Positioning Using a TerrainIndependent Dynamic Propagation Model,» de IEEE Global Communications Conference GLOBECOM, Singapur,, 2017.

[27] Internet Engineering Task Force, «Request for Comments 8376: Low-Power Wide Area Network (LPWAN) Overview,» Dublin, 2018.

[28] U. Raza, P. Kulkarni y M. Sooriyabandara, «Low Power Wide Area Networks: An Overview,» IEEE Communications Surveys \& Tutorials, pp. 855-873, 2017.

[29] «Normatividad de las Telecomunicaciones,» Julio 2015. [En línea]. Available: http://bit.ly/2zA92vW. [Último acceso: marzo 2019]. 